\title{
Educación Estomatológica basada en competencias con aplicación a la virtualidad en tiempos de COVID-19
}

Stomatological competency-based education with application to virtuality in times of COVID-19

\author{
Consuelo Marroquín-Soto ${ }^{1, a}$, César-Augusto Padilla-Avalos ${ }^{1, b}$
}

Sr. Editor:

La educación por competencias es el paradigma y enfoque contemporáneo el cual, basado en resultados cuantificables, busca la formación de profesionales con sólidos conocimientos, habilidades y actitudes; con vocación de servicio y con la capacidad de insertarse en el margen de las exigencias laborales (1). En estomatología, la educación por competencias, es un término que en los últimos años se ha ido familiarizando en el entorno, siendo introducido por primera vez por Chambers, quien definió a las competencias como las habilidades esenciales para iniciar la práctica estomatológica, que combina el conocimiento previo con las actitudes profesionales para el desenvolvimiento de manera autónoma (2). Años posteriores, el concepto de competencias se ha ido complementando con: las habilidades, la comprensión y los valores profesionales, que son necesarios para que el egresado inicie la práctica estomatológica de manera independiente (3).

Actualmente, la educación basada en competencias es el método empleado para educar a los estudiantes de estomatología, considerado un medio confiable para evaluar los resultados del proceso enseñanzaaprendizaje, así como del desempeño de los estudiantes. De este modo, no solo proporciona un método para medir los conocimientos y habilidades, sino también para que el estomatólogo desarrolle aptitudes de sensibilidad y cualidades de servicio a su comunidad (4).

Con la llegada de la pandemia del COVID-19, la educación estomatológica migró a la virtualidad, al igual que otras carreras profesionales. Este proceso de adaptación pudo ser realizado debido a la diversidad de herramientas tecnológicas disponibles. De esta manera, las escuelas de estomatología reemplazaron las aulas físicas por un escenario virtual, convirtiéndose en una oportunidad para retomar la educación a distancia y así optimizar los recursos (5).

Meng, et al., elaboraron un estudio y evidenciaron las dificultades que los estudiantes de estomatología han presentado debido a la COVID-19 (6), en su investigación se analizó el proceso educativo en

Facultad de Odontología, Universidad de San Martín de Porres. Lima, Perú.

Cirujana Dentista. Especialista en Rehabilitación Oral. ORCID ID: https://orcid.org/0000-0002-1433-6205

Cirujano Dentista. Maestro en Periodoncia. ORCID ID: https://orcid.org/0000-0002-8436-4113 
la Escuela de Estomatología de la Universidad de Wuhan, China durante el inicio de la pandemia. Se brindaron recomendaciones sobre las medidas de bioseguridad y el control de infecciones en el ámbito clínico; se recomendó la implementación de videoconferencias en tiempo real y el estudio virtual de casos clínicos, con el fin de evitar la exposición de los estudiantes (6).

Por consiguiente, la virtualización de la educación en estomatología lleva a la reflexión de la formación de profesionales de países en vías de desarrollo, generando la interrogante si estamos preparados para digitalizar el plan curricular ya establecido. La adaptación del proceso enseñanza- aprendizaje a la virtualidad ha sido progresiva, en la búsqueda de conservar el enfoque de la educación basada en competencias. Esta tarea ha involucrado tanto instituciones como a los mismos protagonistas: docentes y estudiantes. En ese sentido, las facultades estomatológicas deben replantear el currículo educativo para que, en la virtualidad, se puedan evaluar las competencias cognitivas, procedimentales y actitudinales de los futuros profesionales en formación.

En la actualidad, se propone la reactivación progresiva de los programas académicos a la semi presencialidad para alcanzar el desarrollo de las competencias procedimentales que son esenciales en el ejercicio estomatológico.

Conflicto de intereses: Los autores no tienen conflicto de interés con este informe.

Aprobación de ética: No requiere.

Financiamiento: Ninguno.

Contribuciones de los autores: Todos los autores contribuyeron a este manuscrito.

\section{Correspondencia:}

Consuelo Marroquín Soto

Correo electrónico: marroquinconsuelo@gmail.com

\section{REFERENCIAS BIBLIOGRÁFICAS}

1. Alkhodary MA, Farah RI, Ghobashy AI. Competencybased education in undergraduate clinical prosthodontics: A paradigm shift in training. J Competency-Based Educ. 2020; 5(3). Doi: 10.1002/ cbe2.1220

2. Chambers DW. Toward a competency-based curriculum. J Dent Educ. 1993;57(11):790-3. Doi: 10.1002/j.0022-0337.1993.57.11.tb02806.x

3. Boyd MA, Gerrow JD, Chambers DW, Henderson BJ. Competencies for dental licensure in Canada. J Dent Educ. 1996;60(10):842-6.

4. American Dental Education Association. Competencies for the New General Dentist. The Voice of Dental Education; 2008. (Citado 5 de septiembre de 2021). Disponible en: https://www.adea.org/about_adea/ governance/pages/competencies-for-the-new-generaldentist.aspx

5. Chavarría-Bolaños D, Gómez-Fernández A, DittelJiménez C, et al. E-Learning in Dental Schools in the Times of COVID-19: A Review and Analysis of an Educational Resource in Times of the COVID-19 Pandemic. Odovtos Int J Dent Sci. 2020;22(3):69-86. Doi: $10.15517 / \mathrm{ijds} .2020 .41813$

6. Meng L, Hua F, Bian Z. Coronavirus Disease 2019 (COVID-19): Emerging and Future Challenges for Dental and Oral Medicine. J Dent Res. 2020;99(5):4817. doi: $10.1177 / 0022034520914246$

Recibido : 09-09-2021

Aceptado : 09-09-2021 